\title{
Géolinguistique
}

15 | 2015

La géographie linguistique au Brésil

\section{Les atlas linguistiques de Sergipe : ALS et ALS II}

Sergipe Linguistic Atlases: ALS and ALS II

Ana Regina Torres Ferreira Teles, Marcela Moura Torres Paim et Silvana Soares Costa Ribeiro

\section{OpenEdition}

\section{Journals}

Édition électronique

URL : http://journals.openedition.org/geolinguistique/624

DOI : $10.4000 /$ geolinguistique.624

ISSN : 2650-8176

\section{Éditeur}

UGA Éditions/Université Grenoble Alpes

\section{Édition imprimée}

Date de publication : 1 décembre 2015

Pagination : 83-100

ISBN : 978-2-84310-317-9

ISSN : 0761-9081

\section{Référence électronique}

Ana Regina Torres Ferreira Teles, Marcela Moura Torres Paim et Silvana Soares Costa Ribeiro, « Les atlas linguistiques de Sergipe : ALS et ALS II », Géolinguistique [En ligne], 15 | 2015, mis en ligne le 15 février 2019, consulté le 30 octobre 2020. URL : http://journals.openedition.org/geolinguistique/624 ; DOI : https://doi.org/10.4000/geolinguistique.624 


\title{
Les atlas linguistiques de Sergipe : ALS et ALS II
}

\author{
Ana Regina Torres Ferreira Teles \\ Marcela Moura Torres Paim \\ Silvana Soares Costa Ribeiro \\ Universidade Federal da Bahia (Brésil)
}

\section{Résumé}

Cet article traite des atlas linguistiques se référant à l'État de Sergipe, l'Atlas Lingüístico de Sergipe (Ferreira et al., 1987) et l'Atlas Lingüístico de Sergipe II (Cardoso, 2005a), des ouvrages qui, du point de vue méthodologique, suivent les pas de l'Atlas Prévio dos Falares Baianos - APFB (Rossi, 1963) et, du point de vue spatial, s'insèrent dans l'aire du «parler baïanais» (Nascentes, 1953). Nous mettrons en évidence : (i) l'historique de sa réalisation; (ii) le réseau de points; (iii) la sélection d'informateurs ; (iv) le questionnaire appliqué; et (v) les innovations que ces deux atlas présentent par rapport à l'APFB. On inclut ici la carte du réseau de points conçue sous un nouveau formatage, dessinée à partir de la cartographie officielle, avec la géo-référence des 15 points qui constituent le réseau de localités des atlas. On présente ainsi une nouvelle carte-base, chaque localité étant associée à un géocode adopté par l'Institut brésilien de géographie et statistique, ce qui rendra possible la liaison des points recherchés au Sergipe à la base de données de l'IBGE. Cette nouvelle configuration cartographique présente une plus grande lisibilité, permettant la réalisation d'études comparatives plus aisées avec les données linguistiques présentes dans les atlas.

\section{Mots-clés}

La géolinguistique au Brésil, atlas linguistiques régionaux, atlas de Sergipe. 


\begin{abstract}
This paper deals with the linguistic atlases regarding the state of Sergipe: the Atlas Lingüístico de Sergipe (Ferreira et al., 1987) and the Atlas Lingüístico de Sergipe II (Cardoso, 2005), works that, from a methodological point of view, follow the steps of the Atlas Prévio dos Falares Baianos - APFB (Rossi, 1953) and, from a spatial point of view, fall into the field of the "speak Bahian" (Nascentes, 1953). We bring considerations on both atlases, highlighting: (i) their realization history; (ii) the points network; (iii) the informants selection; (iv) the utilised questionnaire; and (v) the innovations they represent in relation to the APFB. To this description we add the map of the points network drawed under a new format, prepared on the base of the official cartography with the georeferencing of the 15 points that build the atlases network localities. Thus, we present a new base map where all locations are linked to their respective geocoding adopted by IBGE (Brazilian Institute of Geography and Statistics), which enables to link the ALS points to the IBGE database. This new cartographic configuration allows a greater readability and permits to carry on easier comparative studies with the linguistic data presented in the atlas.
\end{abstract}

\title{
Keywords
}

Geolinguistic in Brazil, regional linguistic atlases, Sergipe atlases.

\section{Les atlas linguistiques analysés}

\subsection{Marquant le point de départ}

Un atlas linguistique est un instrument riche et le fruit d'un travail extensif de la part de ses auteurs. On y trouve la représentation de la variation linguistique, soit éminemment spatiale, soit sociale. Selon Ferreira et al. :

Um atlas lingüístico reúne um conjunto de mapas de um território, mais ou menos vasto, que representam e localizam as realizações dos paradigmas lingüísticos em estudo (de natureza fonética, lexical, morfológica ou sintática), registrando as respectivas variações geográficas ${ }^{1}$. (1996, p. 484)

1. «Un atlas linguistique réunit un ensemble de cartes d'un territoire, plus ou moins vaste, qui représentent et localisent les réalisations des paradigmes linguistiques en étude (de nature phonétique, lexicale, morphologique ou syntaxique), en enregistrant les respectives variations géographiques.» 
Ainsi, le domaine géographique-spatial qu'un atlas linguistique recouvre peut être prédéterminé par des critères de différentes natures. Les atlas linguistiques offrent aussi une meilleure visualisation de la distribution spatiale d'un phénomène linguistique donné, en plus de délimiter son extension. Il est opportun de citer que les atlas ne sont pas un conglomérat de données linguistiques, mais plutôt, selon Ferreira et al. :

Os atlas permitem ainda isolar áreas dialectais, definidas pela concentração de fenômenos lingüísticos idênticos, que, em determinados tipos de mapas, podem ser circunscritos por isoglosas, linhas que marcam o limite geográfico de um fenômeno lingüístico. Um maior número de isoglossas a delimitar uma área determina a sua maior individualidade lingüística.

Por estes motivos, os atlas constituem um instrumento de trabalho indispensável para a Dialectologia e para a História da Língua². (Ibid.)

À partir de ces considérations préliminaires, on remarque qu'un atlas linguistique est un instrument d'extrême importance pour les études linguistiques, car, en plus de sauvegarder la mémoire sociolinguistique d'un peuple (documentation de l'histoire de la langue), il peut être un puissant instrument pour les politiques linguistiques (principalement en ce qui concerne les politiques d'enseignement). Incontestablement, un atlas est un trésor très précieux pour de nombreux domaines de la science.

La publication des atlas linguistiques régionaux, concernant le portugais du Brésil, se configure comme un reflet des mouvements déclenchés à partir de 1952, quand on a institué, à partir du décret $n^{\circ} 30.643$, la Maison de Rui Barbosa. À cette occasion, on a établi comme principale tâche de sa Commission de philologie, l'élaboration de l'atlas linguistique du Brésil (voir Ferreira \& Cardoso, 1994, p. 37).

Devant cette proposition, des experts de la langue, comme Serafim da Silva Neto, Celso Cunha et Antenor Nascentes, se consacrent à l'élaboration des atlas par région, en tenant compte de la réalité socio-économique du Brésil de cette époque et du manque de maturité des chercheurs brésiliens, dans le cadre de la recherche dialectologique. De telles entreprises devraient avoir comme paramètre la possibilité d'inter-comparaison, visant à la description de la réalité nationale, comme un tout.

2. «Les atlas permettent encore d'isoler des aires dialectales, définies par la concentration de phénomènes linguistiques identiques, qui, en certains types de cartes, peuvent être circonscrits par des isoglosses, des lignes qui marquent la limite géographique d'un phénomène linguistique. Un plus grand nombre d'isoglosses visant à délimiter une aire détermine sa plus grande individualité linguistique. Pour toutes ces raisons, les atlas constituent un instrument de travail indispensable pour la dialectologie et pour l'histoire de la langue.» 
Le scénario de cette recherche est constitué par les atlas linguistiques concernant l'État de Sergipe, l'Atlas Lingüístico de Sergipe - ALS (Ferreira et al., 1987) et l'Atlas Lingüístico de Sergipe II - ALS II (Cardoso, 2005a), des œuvres qui, du point de vue méthodologique, suivent les pas de l'Atlas Prévio dos Falares Baianos (APFB), d'après Nelson Rossi et les collaboratrices Carlota Ferreira et Dinah Isensee, et qui fut le premier atlas linguistique produit en terres brésiliennes, élaboré et publié entre 1960 et 1963. Il recouvre tout l'État de Bahia, avec un réseau de 50 localités, qui se distribuent sur différentes aires géographiques et culturelles. Cette œuvre est constituée de deux volumes : le premier, qui comprend les cartes, en feuillets détachés, et le second, relié, contenant l'introduction, le questionnaire commenté et la liste des réponses transcrites. L'APFB concerne les champs sémantiques de l'agriculture, l'anatomie et la physiologie humaine, la cuisine et l'alimentation, la géographie et l'astronomie et comporte 209 cartes, dont 11 sont introductives et 198 linguistiques. En partant de l'analyse exhaustive des sondages préliminaires, on a élaboré un Extrait de questionnaire avec 182 questions (bien que la numérotation se termine à 164, quelques-unes de ces questions sont subdivisées et indexées avec les lettres A, B , C et D) et deux sujets par point ont été interrogés. Malgré l'intention d'insérer, dans toutes les localités, des informateurs des deux sexes et d'âges différents, il n'a pas été possible d'étudier, de façon systématique, la variation diasexuelle. Il est important de mentionner que l'APFB a mis en évidence des traits phonétiques, lexicaux et sémantiques d'importante représentativité.

\subsection{Sergipe : un État à deux atlas}

En empruntant le titre utilisé par Cardoso (2005b), on présente ci-après les caractéristiques de chacun des atlas en question. L'État de Sergipe, un des neuf États qui intègre la région Nordeste du Brésil, se limite à l'est avec l'océan Atlantique; à l'ouest et au sud, par l'État de Bahia; et au nord par l'État d'Alagoas, tout cet espace étant limité par le fleuve São Francisco.

Selon l'Institut brésilien de géographie et statistique (IBGE), le Sergipe présentait une population de 2068017 habitants au recensement 2010, et recouvre une superficie de $21915,116 \mathrm{~km}^{2}$ (le plus petit État brésilien), présentant une densité démographique de 94,35 habitants $/ \mathrm{km}^{2}$ et composé de 75 municipalités. L'Atlas Lingüístico de Sergipe, en ce qui concerne le recueil de données et la préparation des cartes, aurait pu être imprimé dès 1973 ; cependant, à cause de difficulté de financement, sa publication n'a été concrétisée qu'en 1987. Le travail a été mené par le groupe de chercheurs de Bahia : Carlota Ferreira, Jacyra Mota, Judith Freitas, Nadja Andrade, Suzana Cardoso, Vera Rollemberg et Nelson Rossi, dont quelques-uns ont 
fait partie de l'équipe responsable de l'APFB. L'atlas est consacré aux champs sémantiques de la terre, de l'homme, des animaux et des plantes et comporte 180 cartes linguistiques que l'on retrouve dans l'APFB. Dans l'Atlas Lingüístico de Sergipe, il existe encore une série de cartes conjointes Bahia-Sergipe, avec des données de Bahia qui ne figuraient pas dans l'APFB : ces cartes sont désignées comme cartes BASE.

L'ALS a un réseau de 15 localités, comprenant 30 informateurs d'âge compris entre 30 à 65 ans, des deux sexes, analphabètes (et aussi semianalphabètes).

Comme le soulignent Saramago et Cardoso (2010), l'Atlas Lingüístico de Sergipe montre une évolution du point de vue méthodologique par rapport à l'APFB, à savoir : a) l'application d'enquêtes préliminaires enregistrées dans les 15 localités qui ont constitué le réseau de points; b) une plus grande amplitude du questionnaire définitif par rapport à celui appliqué à Bahia ; c) la formulation écrite sur le questionnaire même, de la façon de poser la question sur l'item, avec l'objectif d'assurer une plus grande homogénéité aux enquêtes; d) l'inclusion, à chaque point, d'informateurs des deux sexes, en procédant à l'identification systématique de l'informateur féminin comme $A$ et de l'informateur masculin comme $B$, ce qui a permis le contrôle cartographique de cette variable sociolinguistique.

La décision de réaliser un atlas de l'État de Sergipe est due à la collaboration de personnes pour la logistique inhérente aux enquêtes de terrain et cela a aussi permis, du point de vue académico-scientifique, d'accorder un regard particulier sur l'intégration de l'aire des parlers baianais (Nascentes, 1953). Comme l'affirme Cardoso :

Sergipe se apresentava, naquele então, como uma área possível de ser recoberta por uma investigação sistemática e de natureza geolingüística, com uma rede de pontos muito mais adensada do que a da Bahia, e se constitui, segundo a divisão de Nascentes (1953), numa das partes integrantes do que ele classifica e define como "falar baiano", o que iria ampliar o conhecimento empírico dessa área linguística, permitindo, assim, um melhor entendimento do que afirmara Nascentes ${ }^{3}$. (2005, p. 105-106)

3. «Sergipe se présentait, à cette occasion, comme une aire passible d'être recouverte par une investigation systématique et de nature géolinguistique, avec un réseau de points beaucoup plus dense que celui de Bahia, et se constitue, selon la division de Nascentes (1953), comme une des parties intégrantes de ce qu'il classe et définit comme «parler baianais », ce qui amplifierait la connaissance empirique de cette aire linguistique, permettant ainsi une meilleure compréhension de l'affirmation de Nascentes.» 
L'Atlas Lingüístico de Sergipe II a d'abord été présenté par Suzana Cardoso, en 2002, comme thèse de doctorat à l'Université fédérale de Rio de Janeiro, et publié trois ans après. Le but de cet atlas était de donner suite à celui publié en 1987, utilisant les données collectées entre 1967 et 1968, mais non exploitées, d'un champ sémantique : l'homme. Comme le premier atlas de Sergipe, ce dernier est aussi bidimensionnel, puisqu'il focalise cartographiquement deux variables : diatopique et diasexuelle. Il faut aussi préciser que l'Atlas Lingüístico de Sergipe II peut être défini comme un atlas de seconde génération (Cardoso, 2005b, p. 116) puisque, outre le registre géolinguistique, il présente aussi l'interprétation des phénomènes observés qui fait ressortir des aspects diatopiques et sociolinguistiques, comme l'explique Cardoso :

[...] o ALS-II traz considerações sobre (i) aspectos linguístico-antropológicos, com a abordagem das denominações para as principais refeições do dia, ou da presença de zoomorfismos; (ii) implicações diagenéricas; (iii) a constituição do léxico, com destaque para criações neológicas e de ampliação de sentido, brasileirismos, relação som-sentido; (iv) arcaísmos; (v) e, como é de se esperar, estudos de natureza areal ${ }^{4}$. (2005b, p. 125)

Les cartes de l'Atlas Lingüístico de Sergipe II sont onomasiologiques et les données enregistrées sont rassemblées en indices des réponses transcrites et indice onomasiologique. Cet atlas contient aussi un CD avec un petit échantillon sonore de chacun des points du réseau, se présentant comme un instrument complémentaire à la connaissance de la région, surtout concernant les aspects prosodiques.

Dans l'Atlas Lingüístico de Sergipe, les 167 cartes se distribuent comme suit : (i) 11 cartes introductives; (ii) 156 cartes sémantico-lexicales et phonétiques dans lesquelles sont incluses neuf cartes-résumé Sergipe (fig. 1) et dix cartes Bahia-Sergipe (fig. 2). Les notes figurent sur le verso des cartes.

L'Atlas Lingüístico de Sergipe II, quant à lui, possède 108 cartes, dont trois sont introductives et les autres sémantico-lexicales. Les cartes de l'ALS II portent l'indication des informateurs représentée par des symboles. À la différence de l'ALS, il ne présente pas la transcription phonétique sur le recto de la carte, mais sur le verso, avec les notes. Complémentairement,

4. «[...] l'ALS-II présente des considérations sur (i) des aspects linguistico-anthropologiques, avec l'approche des dénominations pour les principaux repas du jour, ou de la présence de zoomorphismes; (ii) des implications diagénériques; (iii) la constitution du lexique, en mettant en évidence les créations néologiques et d'amplification du sens, des "brésilianismes", rapport son-sens; (iv) archaïsmes; (v) et, comme il fallait s'y attendre, des études relatives aux aires.» 
au recto, les cartes présentent, systématiquement, un graphique indiquant la variation diagénérationelle et sur quelques-unes figurent des images des objets recherchés (fig. 3).

Les deux atlas consacrés à l'État de Sergipe offrent donc un ensemble de 261 cartes linguistiques auxquelles s'ajoutent 14 cartes contenant des informations de nature historico-géographique.

Du point de vue méthodologique, les atlas présentent quelques aspects communs : ils embrassent tout l'État de Sergipe, possèdent des corpus collectés auprès de 30 informateurs pour un réseau de 15 points d'enquêtes. La principale différence consiste dans la forme adoptée pour la représentation cartographique, dans l'inclusion d'un indice onomasiologique et d'un chapitre destiné au commentaire des cartes.

\subsubsection{Le questionnaire}

Se basant sur le questionnaire utilisé pour l'atlas de Bahia, mais élargi considérablement, le questionnaire de Sergipe est constitué d'un ensemble de 687 questions qui ont générées d'importantes données donnant lieu à de nombreuses publications, des communications, des réunions scientifiques, des masters et à la thèse de doctorat Atlas Linguiístico de Sergipe II, élaborée avec un grand nombre de données inédites.

Avant d'aboutir au questionnaire définitif, deux autres types de questionnaires ont été appliqués pour l'atlas de Sergipe : ils correspondent à ceux de la phase préliminaire de sondage, qui visaient à l'adéquation du questionnaire déjà appliqué pour l'élaboration de l'APFB, à la nouvelle aire recherchée. Le questionnaire définitif présente la formulation des questions, élaborée après l'application des deux tests de sondage réalisés. Ce procédé méthodologique a permis d'assurer une meilleure uniformité dans le recueil de données. Ces dernières ont été collectées par cinq enquêteurs (un homme et quatre femmes), chercheurs à l'Université fédérale de Bahia, qui ont participé à toutes les phases de réalisation du projet de l'ALS, depuis la conception et l'implémentation jusqu'à la phase d'analyse des données et la cartographie.

Les enquêtes ont été enregistrées in loco sur des bandes magnétiques et transférées ensuite sur des supports plus modernes. Cela a permis de consulter les données collectées pour l'ALS et d'effectuer des études complémentaires, ainsi que d'accéder aux données encore inédites dans l'ALS II.

\subsubsection{Les informateurs}

Les 30 informateurs étaient nés dans la localité de l'enquête, ainsi que leurs parents : ils ont connu peu de déplacements pour des aires distinctes de celles où ils sont nés, ce qui révèle donc la basse mobilité des sujets choisis. 
En général, les personnes interrogées étaient en majorité des analphabètes (21) ou des semi-analphabètes (8) : une seule était scolarisée. La tranche d'âge se situe entre 35 et 51 ans : trois informateurs sont plus âgés (deux de 65 ans et un de 59 ans) et, un seul, plus jeune (30 ans). Du point de vue de la variable diasexuelle, deux informateurs par localité ont été interrogés, distribués équitablement entre les deux sexes. Dans l'introduction de l'Atlas Lingüístico de Sergipe II, l'auteure présente un tableau comparatif des âges des informateurs : bien qu'il n'y ait pas eu de critères définissant les tranches d'âges lors du recueil de données, l'auteure signale que le profil qui se présente permet d'établir trois groupes : (i) groupe d'âge 1 - 30 à 39 ans (11 informateurs); (ii) groupe d'âge $2-40$ à 48 ans (13 informateurs) et (iii) groupe d'âge 3 à partir de 50 ans (6 informateurs).

Concernant les professions, selon Mota (1998, p. 81), les informateurs, dans leur majorité, se sont déclarés agriculteurs/paysans. Pour les autres professions, quatre hommes se sont déclarés pêcheurs et cinq femmes femmes au foyer : les autres exercent les professions de barbier, laboureur, porteur, charretier, roulier, boucher, maçon, ouvrier manœuvre et bouvier. Parmi les femmes, on relève les métiers de brodeuse, charuteira (ouvrière qui fait des cigares), couturière, marchande de quatre saisons et lavandière.

\subsubsection{Le réseau}

En ce qui concerne la constitution de son réseau, l'ALS présente un trait commun à l'APFB : l'étude systématique et détaillée de chacune des municipalités qui intègrent l'État. À ce propos, ont été pris en compte l'ancienneté de la localité, le degré d'isolement, la formation historique, les caractéristiques culturelles, la position dans le contexte des municipalités de l'État et les références d'ordre linguistique, entre autres. Tenant compte de tels critères, ont été retenus 15 points recouvrant les différentes microrégions, dont la distribution est présentée dans le sous-item 2 (voir tableau 1 - Réseau de points de l'ALS). La numérotation adoptée est aussi reliée à l'APFB et son identification débute par le numéro 51. Par rapport au réseau de points, Saramago et Cardoso expliquent que ces localités, sauf le point 65 , étaient, à l'époque de la documentation, des sièges de municipalité. Curralinho, l'unique village représenté, fait encore partie de la municipalité de Poço Redondo, au bord du fleuve São Francisco. Selon les auteurs en question :

O principal meio de comunicação, responsável pela interligação entre as localidades, era o rodoviário. Dentre todas há, porém, de destacar-se a dificuldade de acesso a Curralinho, provido - convém que se repita, àquela época - apenas de uma estrada de natureza carroçável, permitindo trânsito de jipe ou caminhonete do tipo rural. Aos pontos situados à margem do rio São Francisco -Brejo Grande (61), Propriá (62), Gararu (64) e 
Curralinho (65) - se acrescenta a comunicação fluvial, feita por barcos de maior ou menor velocidade, bastante utilizada pela população dessa área ribeirinha ${ }^{5}$. (2010, p. 128-129)

\section{La géo-référence de l'ALS}

Du point de vue cartographique, l'Atlas Lingüístico de Sergipe donne une représentation simplifiée de l'espace et n'offre pas de grands détails géographiques d'une aire déterminée, mise à part la représentation de ses contours. De cette façon, les cartes introductives (cartes I à XI) sont, en réalité, des cartogrammes qui fournissent des informations sur les microrégions de Sergipe : sur les noms officiels, régionaux et anciens des localités; sur leur population en 1950, 1960 et 1970; sur le nom des villages auxquels elles ont appartenu du point de vue administratif; sur les activités économiques plus importantes de chaque localité (d'après les informateurs); les noms des enquêteurs et des transcripteurs de chaque enquête. Dans l'Atlas Lingüístico de Sergipe II, bien que les cartes introductives (cartes I à III) présentent plus d'informations que celles de l'atlas précédent (des éléments d'hydrographie, réseau routier et escales), il manque quand même les éléments principaux : l'orientation et la localisation.

En outre, dans les deux atlas l'identification des localités se fait par des toponymes respectifs, ce qui pourrait impliquer des positionnements équivoques pour de futures études, principalement quand il y a une altération des propres toponymes ou de leur dépendance politico-administrative. La géo-référence des localités implique l'association de chaque localité à un numéro dénommé géocode, défini et contrôlé par l'IBGE (Institut brésilien de géographie et statistique) et unique pour chaque localité du territoire brésilien. Il fonctionne comme un numéro d'identité, écartant ainsi toute ambiguïté dans son identification.

Un autre avantage à signaler, c'est que la géo-référence permet le lien des points recherchés avec la base de données de l'IBGE, assurant ainsi un contrôle total par rapport à l'identification des localités à n'importe quel moment.

5. «Le principal moyen de communication, utilisé relier les localités était la route. Parmi toutes les difficultés, il y a cependant l'accès à Curralinho, qui n'avait - il faut le répéter, à cette époque-là - qu'une route carrossable permettant le transit de jeep ou de camionnette de type rural. Aux points situés au bord du fleuve São Francisco - Brejo Grande (61), Propriá (62), Gararu (64) et Curralinho (65) - on ajoute la communication fluviale, faite par des bateaux de plus ou moins grande vitesse, très utilisée par la population de cette zone riveraine.» (Saramago \& Cardoso, 2010 ; p. 128-129) 
La carte du réseau des points conçue sous ce nouveau format, élaborée sur une base cartographique officielle, avec la géo-référence des 15 points d'enquêtes, se présente ainsi comme une nouvelle carte-base sur laquelle pourront être enregistrées aussi bien les réponses des cartes originales, que de nouvelles études, sans compter la possibilité d'associer des localités aux données socio-économiques respectives résultant des recensements et d'autres études de cette nature.

Cette nouvelle configuration cartographique présente une plus grande lisibilité et rend possible la réalisation d'études comparatives plus aisées avec les données linguistiques présentes dans les atlas.

Pour construire la nouvelle carte-base, on a cherché à identifier, sur la base des données ${ }^{6}$ de l'IBGE, les localités présentées sur les cartes et à vérifier si leurs dénominations étaient en conformité avec les toponymes officiels enregistrés. Une fois la recherche commencée, on a constaté que, même si les unités politico-administratives étaient pratiquement les mêmes que celles d'aujourd'hui, la subordination politico-administrative a été substantiellement altérée par le fait que le Brésil a adopté une nouvelle division territoriale à partir de $1989^{7}$.

Ainsi, dans l'introduction de l'ALS, concernant les localités enquêtées et dans l'ALS II, au chapitre 1, le point 51 est écrit Santa Luzia do Itanhi, mais dans les documents de l'IBGE, depuis le recensement de 1950 jusqu'à celui de 2010, on relève la graphie Santa Luzia do Itanhy. Cependant, dans les cartes des localités des deux atlas, le toponyme a été réduit à Santa Luzia. Malgré l'apparente innocuité de ces remarques, elles sont pertinentes dans la mesure où, lorsqu'on travaille avec la base de données, toute différence peut devenir déterminante.

Avec l'adoption du géocode, le point 51 employé dans l'exemple, aura toutes les informations relatives à ce point totalement contrôlées.

Le tableau 1 présente les localités du réseau de points avec les géocodes respectifs, les subordinations administratives (qui sont les mêmes en 1987, en 2005 et en 2015) et les liens avec les divisions territoriales du pays dans les trois moments. Seuls les villages sont géo-référencés par leurs coordonnées géographiques respectives.

6. Base territoriale brésilienne de la bCIMd (Base cartographique intégrée numérisée du Brésil au millionième), v. 2.0.

7. Les zones physiographiques auxquelles sont référencées les localités de l'ALS ont été adoptées par l'IBGE en 1945 et utilisées, parmi d'autres finalités, pour le calcul des données statistiques jusqu'en 1970, quand elles ont été substituées par des microrégions homogènes. À partir de 1989, les informations socio-économiques ont été associées aux micro et mésorégions géographiques. 


\begin{tabular}{|c|c|c|c|c|c|c|c|c|c|c|c|c|c|c|c|c|}
\hline 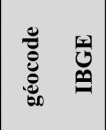 & 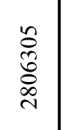 & $\begin{array}{l}\overline{0} \\
\stackrel{5}{0} \\
0 \\
\infty \\
\text { ஸे }\end{array}$ & 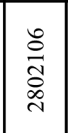 & 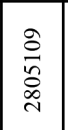 & 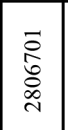 & 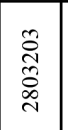 & $\begin{array}{l}\text { Oे } \\
\text { లి } \\
\text { Oे } \\
\text { N }\end{array}$ & 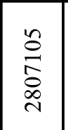 & 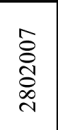 & 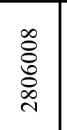 & 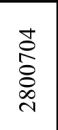 & 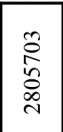 & 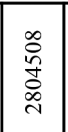 & 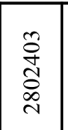 & 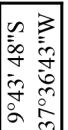 & \\
\hline 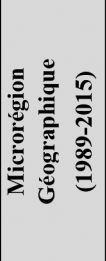 & 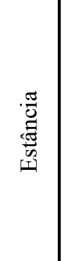 & $\begin{array}{l}\text { 慁 } \\
\text { 。 }\end{array}$ & 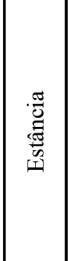 & 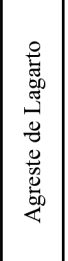 & $\begin{array}{l}\text { 胥 } \\
\text { 莺 }\end{array}$ & 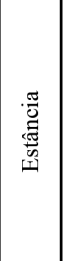 & 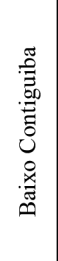 & 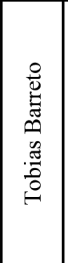 & 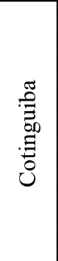 & U & 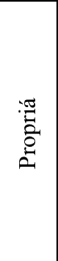 & 莺 & 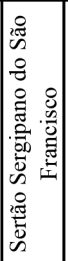 & 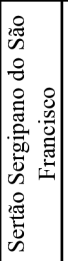 & 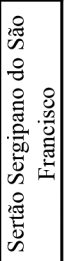 & \\
\hline 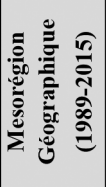 & 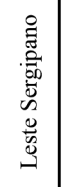 & 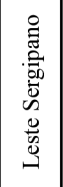 & 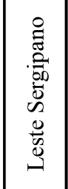 & 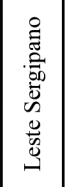 & 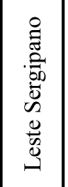 & 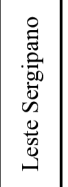 & 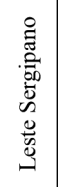 & 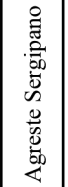 & 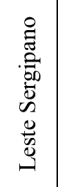 & 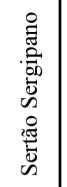 & 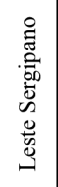 & 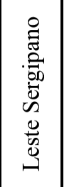 & 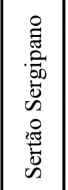 & 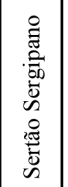 & 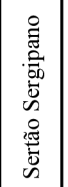 & \\
\hline 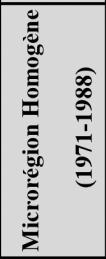 & 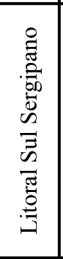 & 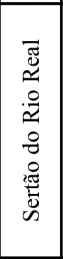 & 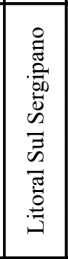 & 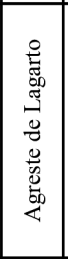 & 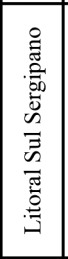 & 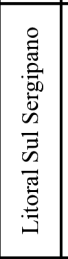 & 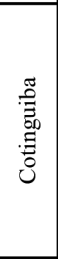 & 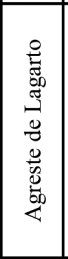 & 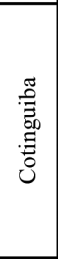 & 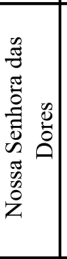 & 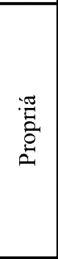 & 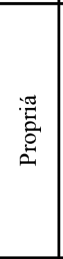 & 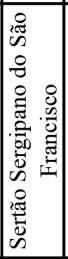 & 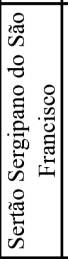 & 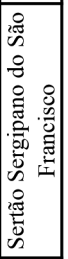 & 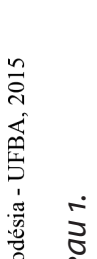 \\
\hline 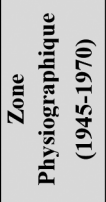 & 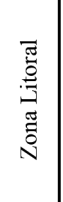 & 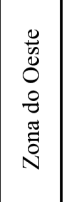 & 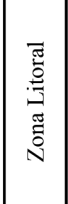 & 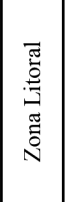 & 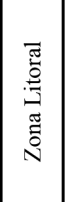 & 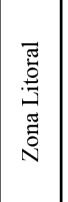 & 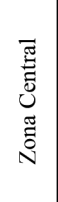 & 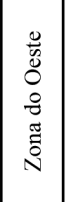 & 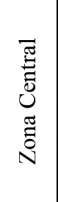 & 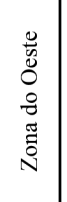 & 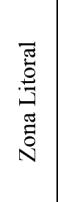 & 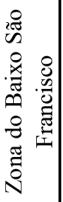 & 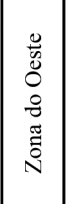 & 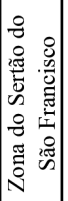 & 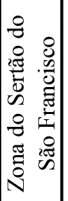 & 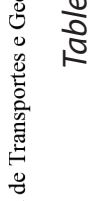 \\
\hline • & 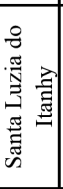 & 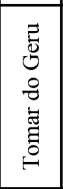 & 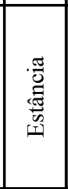 & 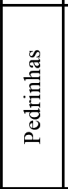 & 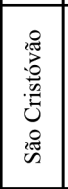 & 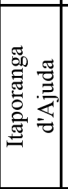 & 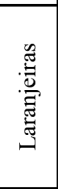 & 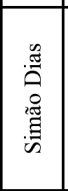 & 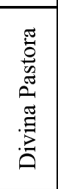 & 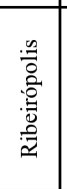 & 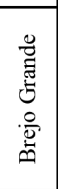 & $\begin{array}{l}\stackrel{0}{2} \\
\stackrel{2}{2} \\
\stackrel{2}{2}\end{array}$ & 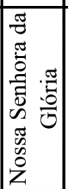 & 렳 & 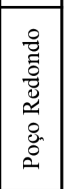 & 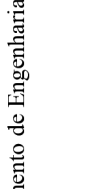 \\
\hline 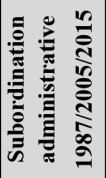 & 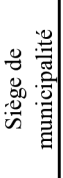 & 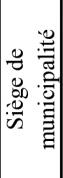 & 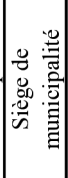 & 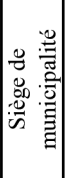 & 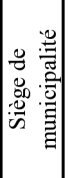 & 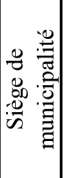 & 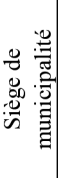 & 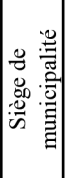 & 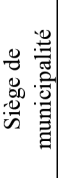 & 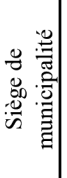 & 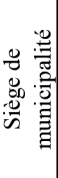 & 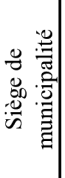 & 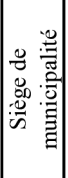 & 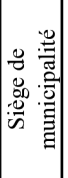 & $\begin{array}{l}\stackrel{8}{5} \\
\stackrel{\Xi}{5}\end{array}$ & 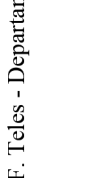 \\
\hline 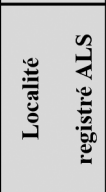 & 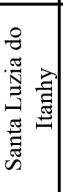 & 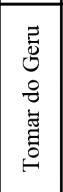 & 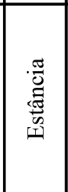 & 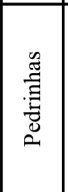 & 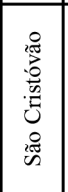 & 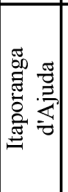 & 莺 & 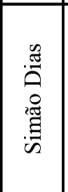 & 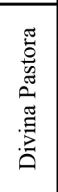 & 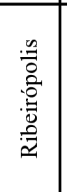 & 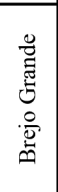 & $\begin{array}{l}\frac{\pi}{2} \\
\stackrel{0}{2} \\
\stackrel{2}{2}\end{array}$ & 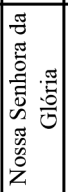 & 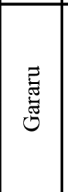 & 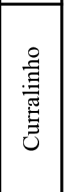 & 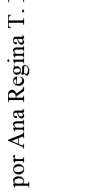 \\
\hline 竞 & $\vec{n}$ & $\tilde{n}$ & $\tilde{n}$ & in & $n$ & $\stackrel{i}{n}$ & $\hat{n}$ & $\stackrel{\infty}{n}$ & ڤิ & $\stackrel{8}{0}$ & $\bar{\sigma}$ & $\hat{\sigma}$ & $\hat{6}$ & ț & $\approx$ & 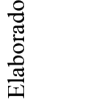 \\
\hline
\end{tabular}


La figure 4 présente la nouvelle carte-base, qui contient tous les éléments cartographiques nécessaires pour la classer comme carte.

\section{Considérations finales}

Les informations linguistiques que contiennent les deux atlas linguistiques de l'État de Sergipe ont permis, durant des décennies, la production de travaux qui ont aidé à la description du Parler baïanais (Nascentes, 1953), ce qui représente un des objectifs établis tant pour l'APFB que pour l'ALS.

En ce qui concerne les aspects linguistiques mis à jour par l'Atlas Lingüístico de Sergipe et par l'Atlas Lingüístico de Sergipe II, il faut mettre en relief l'importance d'entreprendre l'analyse à la recherche de la caractérisation de l'aire que les deux atlas permettent de photographier.

Dans l'introduction de l'Atlas Lingüístico de Sergipe II, deux cartes linguistiques, présentent les tracés d'isoglosses qui révèlent l'aire dialectale proche du fleuve São Francisco, un important fleuve brésilien, connu comme le fleuve de l'intégration nationale. La figure 5 montre les cartes avec la représentation des aires.

Il faut souligner que, comme contribution à la géolinguistique brésilienne, la réédition des cartes originales des atlas linguistiques, comme cela a été fait pour l'ALS et décrit dans l'item 2, est très importante pour la réalisation de travaux de lecture et relecture des atlas publiés. Comme l'ALS, d'autres atlas linguistiques publiés précédemment pourront bénéficier d'une présentation sous une base cartographique officielle, avec la géo-référence des points d'enquête et une nouvelle présentation des données et surtout les informations de nature phonétique, qui, en général, accompagnent l'information lexicale.

\section{RÉFÉRENCES BIBLIOGRAPHIQUES}

CARDoso Suzana Alice Marcelino da Silva, 2005a, Atlas Lingüístico de Sergipe II, Salvador, EDUFBA.

CARdoso Suzana Alice Marcelino da Silva, 2005b, «Sergipe, um estado com dois atlas», dans V. d. A. Aguilera (éd.), A Geolingüística no Brasil, trilhas seguidas, caminhos a percorrer, Londrina, Editora da Universidade Estadual de Londrina, p. 101-135.

CARDoso Suzana Alice Marcelino da Silva, 2010, Geolingüística: tradição e modernidade, São Paulo, Parábola Editorial.

FerReira Carlota et al., 1987, Atlas Lingüístico de Sergipe (ALS), Universidade Federal da Bahia, Salvador, Fundação Estadual de Cultura de Sergipe. 
Ferreira Carlota \& Cardoso Suzana Alice Marcelino da Silva, 1994, A Dialetologia no Brasil, São Paulo, Contexto.

FERreira Manuela Barros et al., 1996, «Variação lingüística, perspectiva dialectológica», dans I. H. Faria et al. (éds), Introdução à Linguística Geral e Portuguesa, Lisboa, Editorial Caminho, S.A., p. 479-502.

Instituto Brasileiro de Geografia e Estatística (IBGE), 2006, bCIMd (Base cartographique intégrée numérisée du Brésil au millionième), v. 2, CD ROM.

IBGE-SIDRA, Sistema IBGE de Recuperação Automática. Disponible sur <www. sidra.ibge.gov.br/bda/territorio> [dernier accès le 15 avril 2015].

Мота Jacyra Andrade, 1998, «Atlas Lingüístico de Sergipe: aspectos metodológicos », dans V. d. A. Aguilera (éd.), A Geolingüística no Brasil, Londrina, Editora da Universidade Estadual de Londrina, caminhos e perspectivas, p. 79-98.

Nascentes Antenor, 1953, O linguajar carioca, Rio de Janeiro, éd. Organização Simões.

Rossi Nelson, 1963, Atlas Prévio dos Falares Baianos (APFB), Ministério de Educação e Cultura, Rio de Janeiro, Instituto Nacional do Livro.

Saramago João \& CArdoso Suzana Alice Marcelino da Silva, 2010, «Atlas Lingüístico de Sergipe. História, metodologia e abordagem dialectométrica», Estudos lingüísticos e literários, $\mathrm{n}^{\circ}$ 41, p. 121-167. 


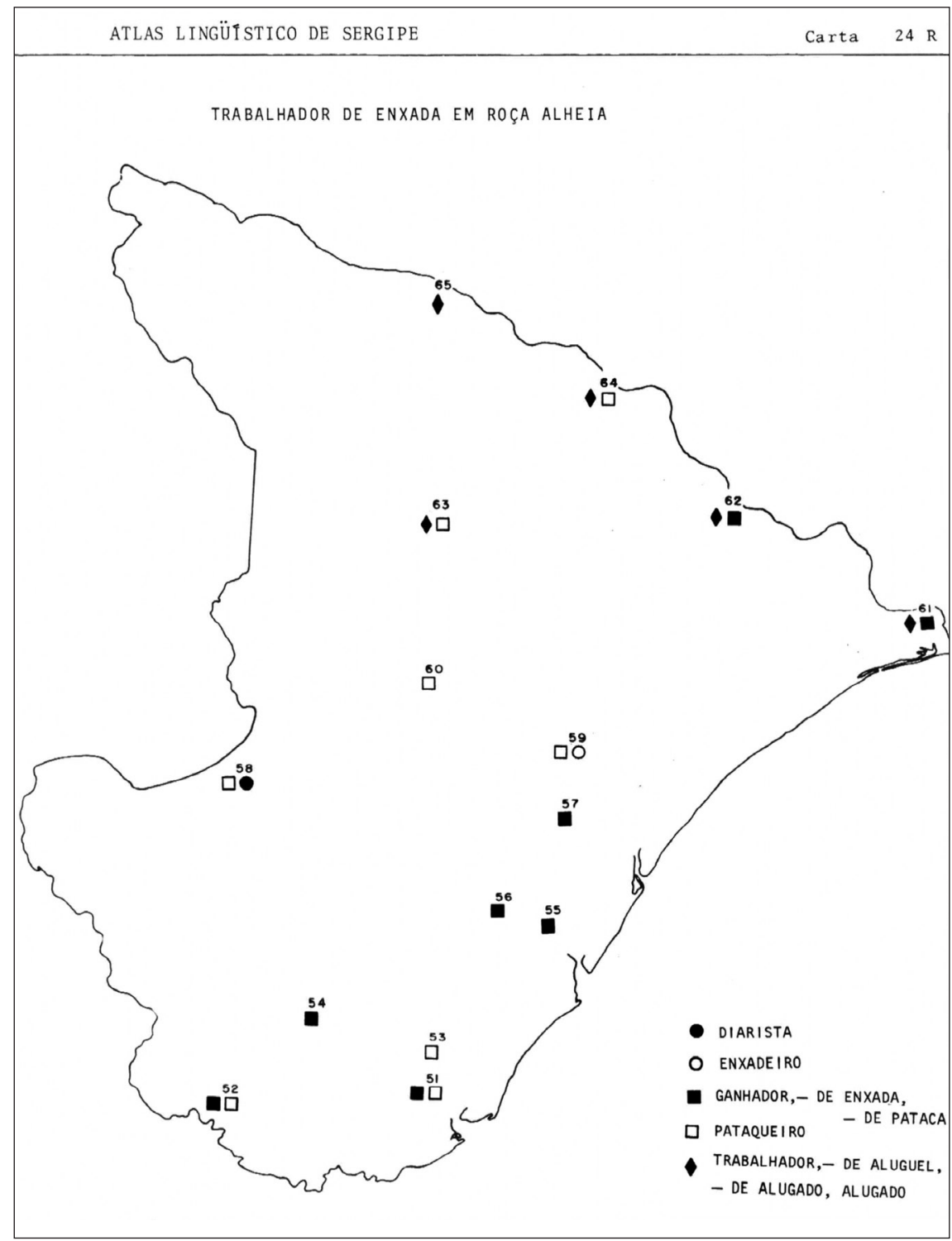

- diarista : journalier

- enxadeiro : bêcheur

- ganhador - de enxada - de pataca : journalier qui travaille avec la bêche.

- trabalhador - de aluguel - de alugado - alugado : paysan embauché pour travailler le champ d'autrui.

Figure 1. - Carte $24 R$ : Travailleur journalier qui laboure le champ d'autrui. Atlas Lingüístico de Sergipe (Ferreira et al., 1987). 


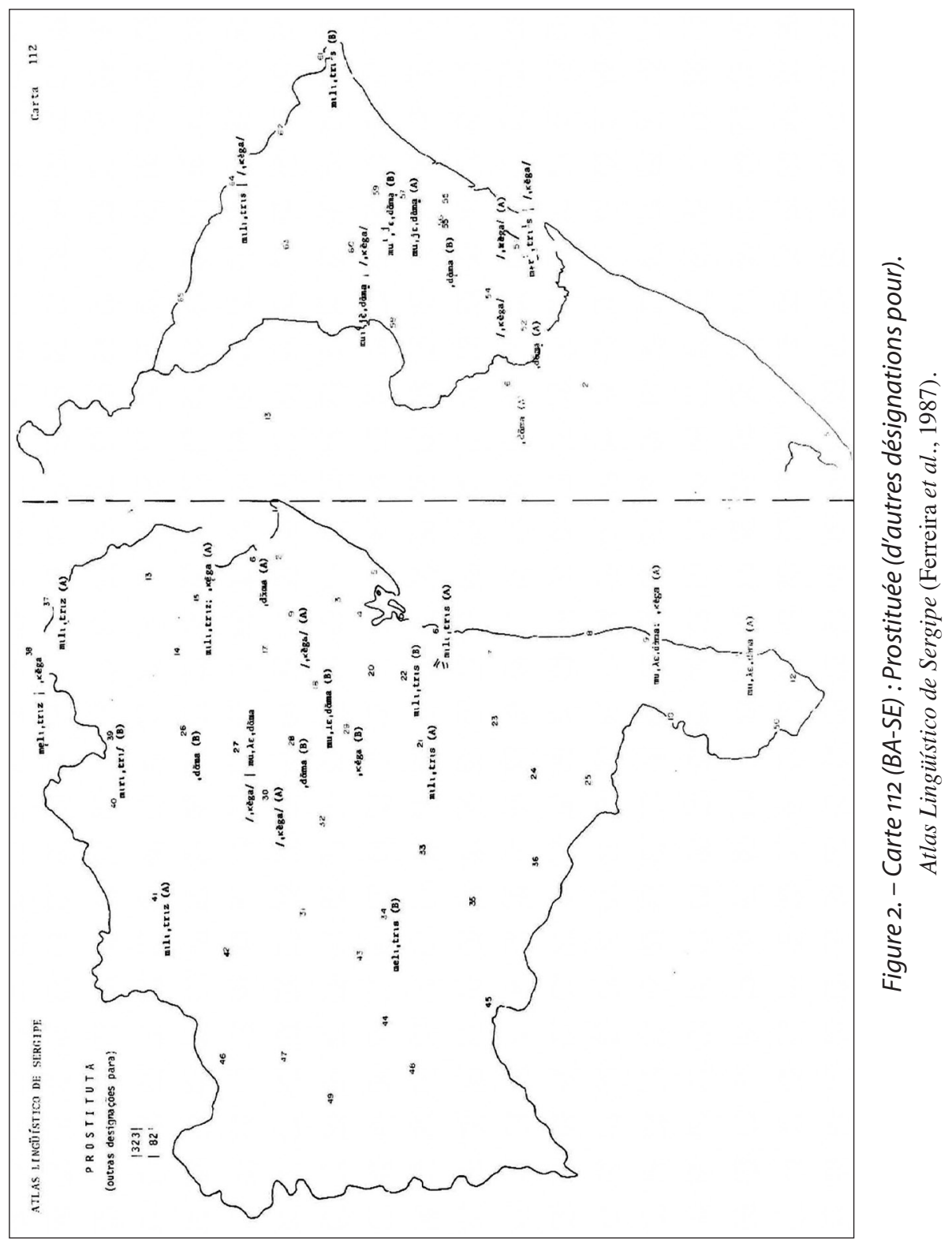


ATLAS LINGÜISTICO DE SERGIPE - II

61

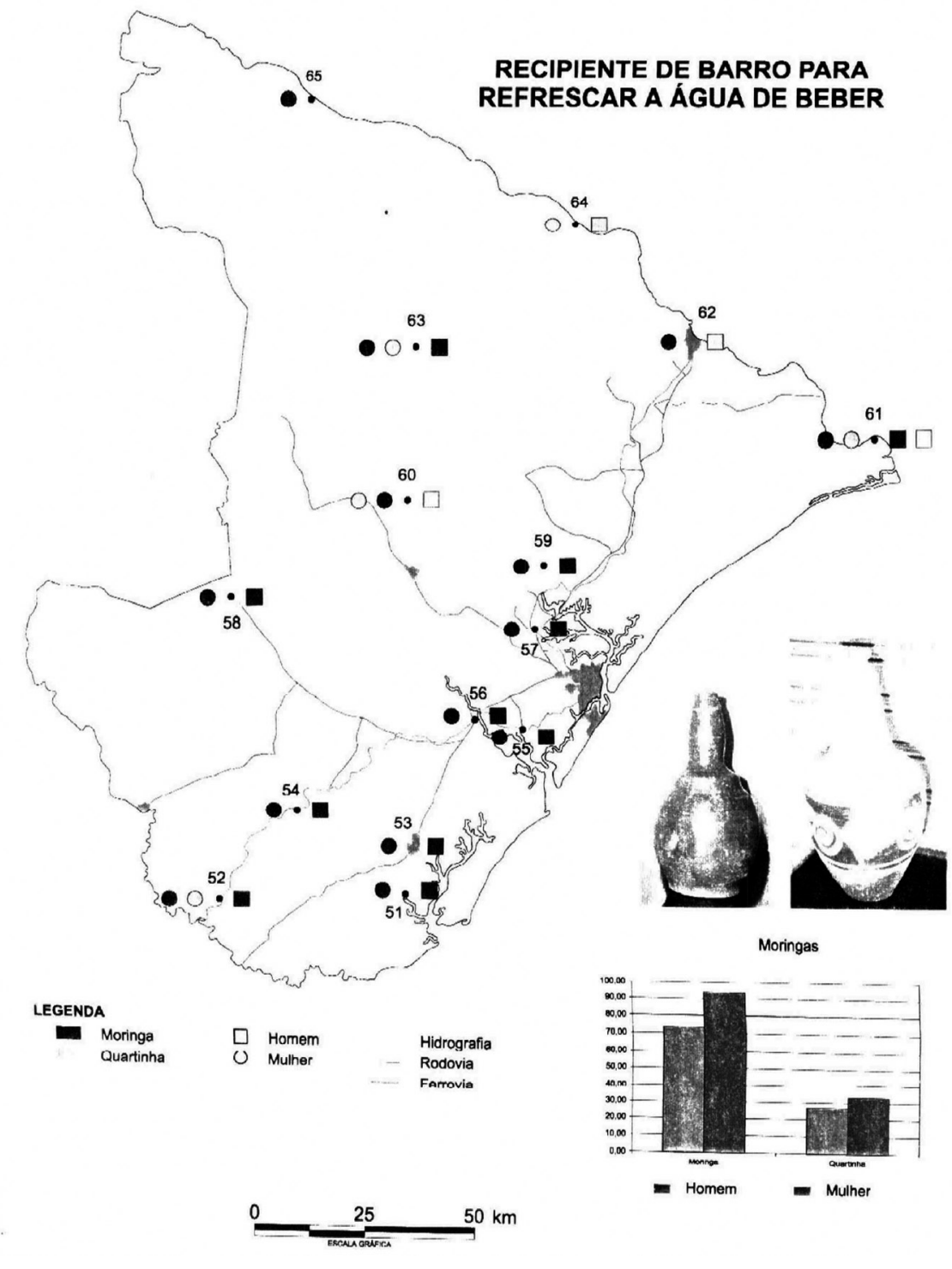

Figure 3. - Carte 61 : Récipient en céramique pour rafraîchir l'eau à boire. Atlas Lingüístico de Sergipe II (Cardoso, 2005a). 


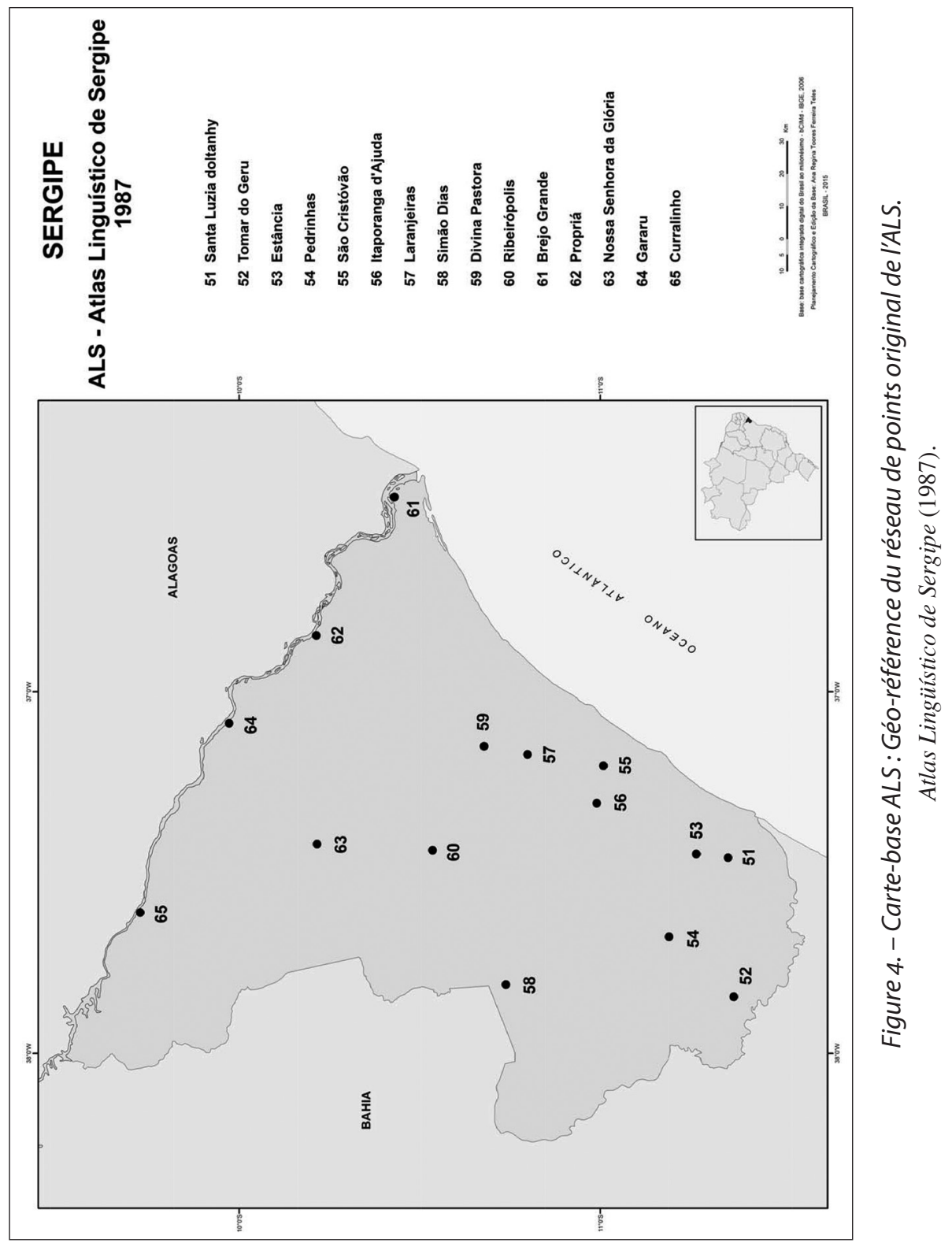




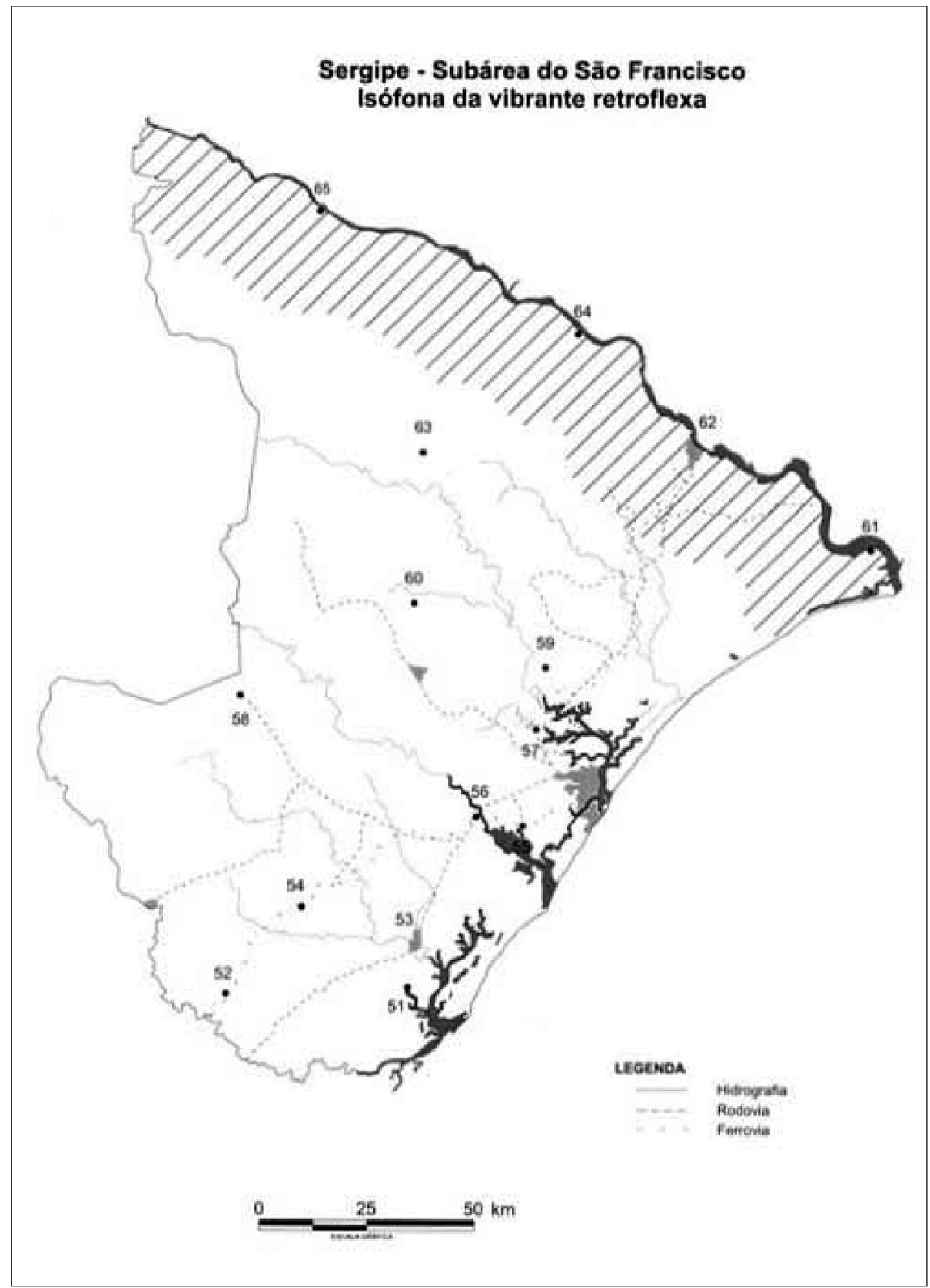

Figure 5. - Sergipe - Sous-aire du São Francisco : Isophone de la vibrante rétroflexe. Atlas Lingüístico de Sergipe II (Cardoso, 2005a, p. 67). 